\title{
Características necessárias para publicação em periódicos de enfermagem: protocolo
}

\section{de scoping review}

Necessary characteristics for publication in nursing journals: scoping review protocol

Características necesarias para la publicación en revistas de enfermería: protocolo scoping review

Rayane Nascimbeni Maldonado

ORCID: https://orcid.org/0000-0002-2344-6983 Universidade Estadual de Londrina, Brasil

E-mail: rayane_nascimbeni@hotamil.com

Helenize Ferreira Lima Leachi

ORCID: https://orcid.org/0000-0002-7792-3407

Universidade Estadual de Londrina, Brasil

E-mail: nizeflima@hotmail.com

Aryane Apolinario Bieniek

ORCID: https://orcid.org/0000-0001-8622-1741

Universidade Estadual de Londrina, Brasil

E-mail: aryanebieniek@gmail.com

Patricia Aroni

ORCID: https://orcid.org/0000-0001-5092-2714

Universidade Estadual de Londrina, Brasil

E-mail: aronipatricia@gmail.com

Renata Perfeito Ribeiro

ORCID: https://orcid.org/0000-0002-7821-9980

Universidade Estadual de Londrina, Brasil

E-mail: perfeitorenata@gmail.com

\section{Resumo}

Introdução: As características presentes em um artigo científico, contribuem para que o mesmo, seja considerado de qualidade e tenha maior chances de publicação. Objetivo: Analisar as evidências científicas disponíveis na literatura sobre as características necessárias para a publicação de artigos científicos em periódicos da área de enfermagem. Método: Protocolo de scoping review fundamentado pelo Joanna Briggs Institute. Pretende responder à seguinte questão: fundamentada pela estratégia População-Conceito-Contexto (PCC): Quais as evidências científicas disponíveis na literatura sobre as características necessárias para a publicação de artigos científicos em periódicos da área de enfermagem? Foram incluídos estudos que abordassem as características relevantes na escrita de artigos científicos em revistas de enfermagem, disponíveis na íntegra, sem delimitação temporal e/ou de idioma. Resultados: A descrição dos achados ocorrerá de forma descritiva, por meio de um quadro, no qual estarão os principais resultados de cada estudo incluído. Conclusão: A redação científica bem como critérios de execução da pesquisa, são fatores determinantes para a publicação em periódicos com fator de impacto mais elevado.

Palavras-chave: Manuscrito; Artigo de revista; Publicação periódica; Redação; Comunicação acadêmica; Enfermagem.

\begin{abstract}
Background: The characteristics present in a scientific article contribute to its being considered of quality and having greater chances of publication. Objective: To analyze the scientific evidence available in the literature on the characteristics necessary for the publication of scientific articles in nursing journals. Method: Scoping review protocol based on the Joanna Briggs Institute. It intends to answer the following question based on the Population-ConceptContext (PCC) strategy: What scientific evidence is available in the literature on the characteristics necessary for the publication of scientific articles in nursing journals? Studies that addressed the relevant characteristics in the writing of scientific articles in nursing journals, available in full, without temporal and/or language limitations, were included. Results: The description of the findings will occur in a descriptive way, through a table, in which will be the main results of each included study. Conclusion: Scientific writing as well as research execution criteria are determining factors for publication in journals with a higher impact factor.
\end{abstract}

Keywords: Manuscript; Journal article; Periodical; Writing; Scholarly communication; Nursing. 


\begin{abstract}
Resumen
Introducción: Las características de un artículo científico pueden contribuir a que sea considerado de calidad y tenga mayores posibilidades de publicación. Objetivo: Analizar la evidencia científica disponible en la literatura sobre las características necesarias para la publicación de artículos científicos en revistas de enfermería. Método de revisión: protocolo de scoping review basado en el Instituto Joanna Briggs. Se pretende dar respuesta a la siguiente pregunta con base en la estrategia Población-Concepto-Contexto (PCC): ¿Qué evidencia científica se encuentra disponible en la literatura sobre las características necesarias para la publicación de artículos científicos en revistas de enfermería? Se incluyeron estudios que abordaron las características relevantes en la redacción de artículos científicos en revistas de enfermería, disponibles en su totalidad, sin limitaciones temporales y/o de idioma. Resultados: La descripción de los hallazgos se producirá de forma descriptiva, a través de una tabla, en la que estarán los principales resultados de cada estudio incluido. Conclusión: La redacción científica y los criterios de ejecución de la investigación son determinantes para la publicación en revistas con mayor factor de impacto.
\end{abstract}

Palabras clave: Manuscrito; Artículo de revista; Publicación periódica; Escritura; Comunicación académica; Enfermería.

\title{
1. Introdução
}

Os periódicos científicos atribuem visibilidade aos avanços das pesquisas, e para que atinjam alto nível de disseminação do conhecimento necessitam atender a critérios de qualidade (Ribeiro \& Marziale, 2018). O maior desafio da gestão editorial é manter a qualidade dos artigos publicados (Marziale, 2017), considerando que se trata de um fator de prestígio dos periódicos científicos.

A originalidade, resultados de estudos comprovados e validados, qualidade da avaliação e gestão editorial que siga boas práticas de divulgação científica são fatores que indicam a função educativa, social e política de uma revista científica (Marziale, 2017). Por isso, a qualidade da ciência e sua apresentação são fatores que podem interferir na aprovação ou rejeição de um manuscrito (Albuquerque, 2009).

Dentre as fragilidades de um manuscrito destaca-se a vagues da introdução, objetivos confusos e desconexos com os resultados e conclusões apresentadas, falta de apropriação da temática, utilização de métodos inadequados, discussão inconsistente na qual não há a contextualização sobre os achados, e linguagem imprópria (Albuquerque, 2009).

Além de que, muitos artigos não conseguem descrever qual o avanço para a ciência na determinada área. Portanto, o que já foi descoberto anteriormente, não se tem a necessidade de ser publicado novamente, pois isso a necessidade do avanço científico presente no manuscrito apresentado.

Destaca-se também que para a efetividade da comunicação cientifica, os resultados das pesquisas precisam apresentar impacto nos processos saúde-doença das populações de outros países, no cenário mundial. Este aspecto tem sido discutido em fóruns, no sentido de promover estratégias para aumentar a visibilidade e consequente internacionalização dos periódicos científicos (Crossetti, 2015). Dessa forma, os periódicos brasileiros levam as pesquisas para o mundo, mostrando o que é feito no Brasil e aumentando o prestígio dos periódicos e pesquisadores à nível mundial.

Atualmente, a rede EQUATOR (Enhancing the Quality and Transparency of Health Research), lista mais de 250 guidelines direcionados à avaliação e redação de estudos de diversos tipos de métodos (Pacheco et al., 2017), no entanto, não há nenhuma revisão de literatura focada nas características gerais necessárias para a publicação, sobretudo, na área da enfermagem, deixando os avaliadores de estudos científicos sem um caminho certo para percorrer. Esse caminho se dá, por meio da experiência e expertise de cada avaliador.

Diante deste contexto, tem-se como objetivo desta scoping review: analisar as evidências científicas disponíveis na literatura sobre as características necessárias para a publicação de artigos científicos em periódicos da área de enfermagem. Para alcançar o objetivo determinado, utilizar-se-á da seguinte pergunta norteadora: "Quais as evidências científicas disponíveis na literatura sobre as características necessárias para a publicação de artigos científicos em periódicos da área de enfermagem?". 


\section{Metodologia}

Trata-se de uma scoping review, seguindo as recomendações metodológicas de revisões propostas pelo Joanna Briggs Institute (Peters et al., 2020). Este tipo de estudo permite o mapeamento dos principais conceitos que apoiam determinada área de conhecimento e identifica as lacunas do conhecimento existentes, bem como, fornece uma visão geral da evidência existente.

Para a execução desta pesquisa, apresenta-se neste protocolo as etapas percorridas: identificação da questão de pesquisa; identificação de estudos relevantes; seleção dos estudos conforme critérios predefinidos, extração de dados e apresentação e discussão dos resultados (Peters et al., 2020).

O fluxo metodológico para seleção de artigos da scoping review está descrito na Figura 1.

Figura 1. Workflow do fluxo de seleção de artigos da scoping review. Brasil, 2021.

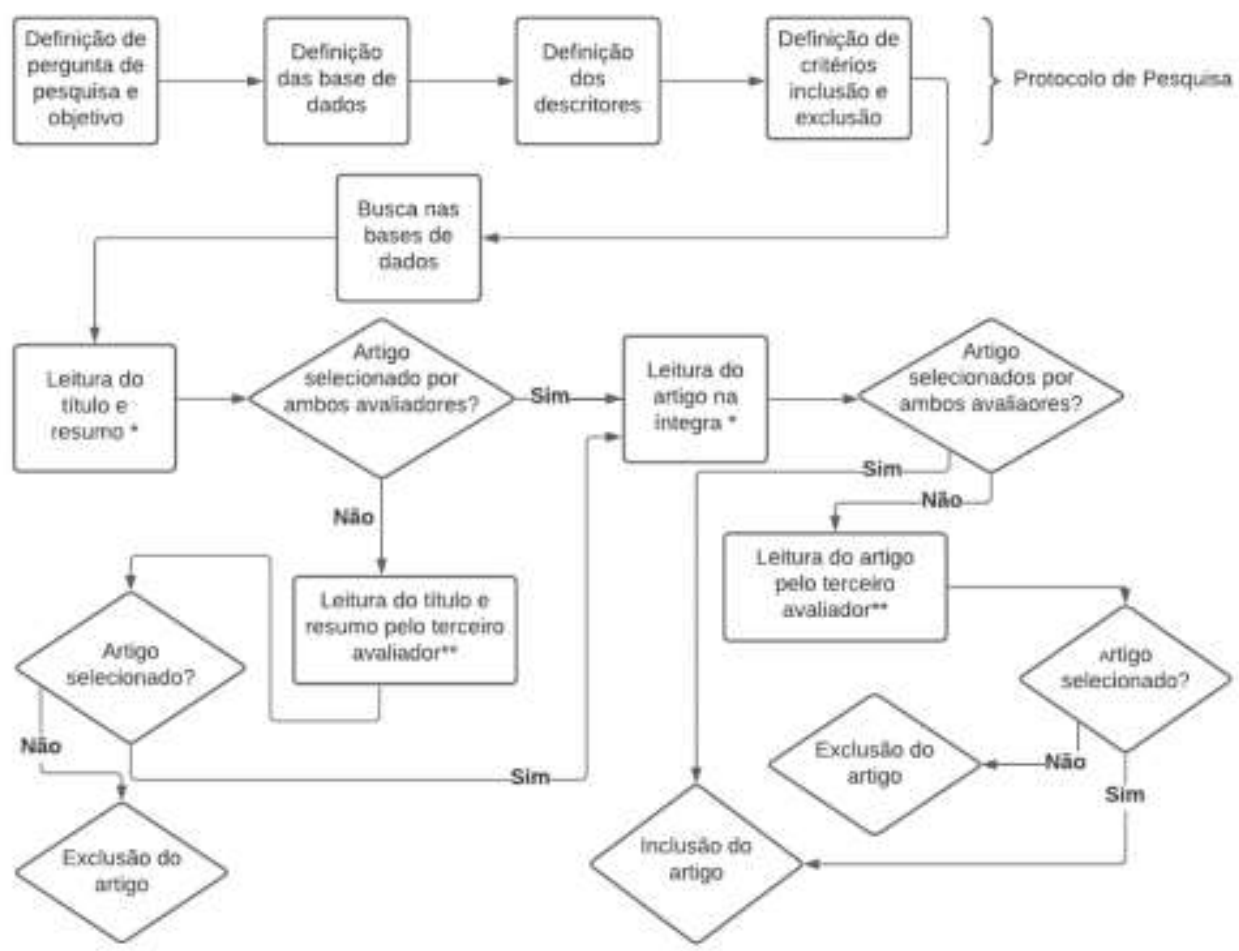

* Realizada por dois avaliadores. **Desempate no caso de divergência de seleção entre os dois primeiros avaliadores. Fonte: Autores (2021).

Considerando que se trata de um estudo bibliográfico, sem envolvimentos de seres humanos, não há necessidade de aprovação por parte de Comitê de Ética em Pesquisa. Contudo, este estudo seguiu os preceitos éticos da escrita de trabalho científico.

\subsection{Estratégia de pesquisa e identificação dos estudos}

A pergunta de pesquisa norteadora desta revisão, foi formulada por meio da estratégia mnemônica PCC (acrônimo para População, Conceito e Contexto) e se apresenta da seguinte forma: "Quais as evidências científicas disponíveis na literatura sobre as características necessárias para a publicação de artigos científicos em periódicos da área de enfermagem?”. Para o primeiro item da estratégia, (P) definiu-se artigo científico/publicações científicas/redação científica; o segundo (C), 
características para publicação e o último (C) revistas de enfermagem.

Foram definidos como critérios de inclusão estudos que abordassem redação científica quanto às características relevantes para publicação em revistas de enfermagem, disponíveis na íntegra, de qualquer idioma e sem delimitação temporal. Foram excluídos estudos secundários.

A busca de dados ocorrerá em nove bases: Literatura Latino-Americana e do Caribe em Ciências da Saúde (LILACS), Medical Literature Analysis and Retrieval System Online (MEDLINE) (via PubMed), SCOPUS, Cumulative Index to Nursing and Allie Health Literature (CINAHL), Web of Science (WOS), Embase, Coordenação de Aperfeiçoamento de Pessoal de Nível Superior (CAPES), Open Grey e Scientific Electronic Library Online (SciELO).

Os descritores utilizados para a busca na LILACS, SciELO e CAPES foram selecionados nos Descritores em Ciência da Saúde (DeCS) da Biblioteca Virtual em Saúde (BVS). Para as bases Medline, Scopus, Open Grey e WOS, os descritores foram selecionados no MeSh Database. As buscas na Embase e CINAHL ocorreram com a utilização dos termos próprios (Quadro 1).

Para as combinações entre os descritores, foram utilizados operadores booleanos AND e OR, sendo AND usado para localizar estudos entre dois temas (intersecção) e OR para sinônimo (Pereira \& Galvão, 2014).

Quadro 1. Descritores e sinônimos utilizados por bases de dados.

\begin{tabular}{|c|c|c|}
\hline Base de Dado & Termos controlados & Termo não controlado \\
\hline $\begin{array}{l}\text { Medline } \\
\text { Scopus } \\
\text { WOS } \\
\text { Open Grey }\end{array}$ & $\begin{array}{l}\text { (Classical Article OR Corrected and Republished Article OR } \\
\text { Newspaper Article OR Journal Article OR Introductory Journal } \\
\text { Article OR Manuscript OR Manuscript, Medical) AND } \\
\text { (Writing OR Publishing OR Guidelines as Topic OR Standards } \\
\text { OR Editorial Policies OR Planning Techniques) AND } \\
\text { (Nursing) }\end{array}$ & Quality writing \\
\hline $\begin{array}{l}\text { LILACS } \\
\text { SciELO } \\
\text { CAPES }\end{array}$ & $\begin{array}{l}\text { (Artigo Clássico OR Artigo Corrigido e Republicado OR } \\
\text { Artigo de Jornal OR Artigo de Revista OR Artigo Introdutório } \\
\text { OR Manuscrito OR Publicação Periódica OR Jornalismo } \\
\text { Científico OR Comunicação e Divulgação Científica) AND } \\
\text { (Redação OR Comunicação Acadêmica OR Editoração OR } \\
\text { Publicação OR Diretrizes OR Políticas Editoriais OR Técnicas } \\
\text { de Planejamento OR Padrões) AND (Enfermagem) }\end{array}$ & - \\
\hline Embase & 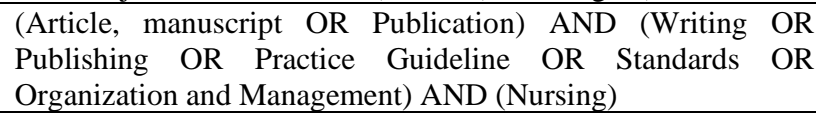 & Quality control \\
\hline CINAHL & $\begin{array}{l}\text { (Articles OR Articles or Journals OR Newspaper articles OR } \\
\text { Journal articles OR Manuscript) AND (Writing OR Publishing } \\
\text { OR Standards OR Planning Techniques OR Writing Strategies } \\
\text { OR Guidelines OR Editorial) AND (Nursing) }\end{array}$ & - \\
\hline
\end{tabular}

Fonte: Autores (2021).

\subsection{Extração de dados}

Conforme exposto na Figura 1, a leitura e seleção dos estudos será realizada por dois revisores de forma independente e às cegas, podendo ter a emissão de parecer de um terceiro revisor, no caso de divergência de seleção entre os dois primeiros. Destaca-se que os critérios de inclusão e exclusão serão obedecidos rigorosamente para a seleção dos estudos, sendo utilizado um roteiro desenvolvido pelos pesquisadores, o qual apresenta o objetivo e a pergunta de pesquisa da scoping review.

O Quadro 2, apresenta o roteiro para extração dos dados pelos revisores, o qual explicita o objetivo e pergunta norteadora deste estudo, base de dados, título do artigo científico, autores, ano de publicação, objetivo, seleção (resposta dicotômica: sim ou não) e motivo de exclusão. Este roteiro foi elaborado no Microsoft Excel® e poderá sofrer alterações, caso algum dos revisores verifique necessidade. Portanto, será realizado um teste piloto antes da fase de extração de dados. 
Quadro 2. Roteiro para extração de dados de scoping review.

\begin{tabular}{|c|c|c|c|c|c|c|}
\hline \multicolumn{7}{|c|}{$\begin{array}{l}\text { Objetivo: Analisar as evidências científicas disponíveis na literatura sobre as características necessárias para a } \\
\text { publicação de artigos científicos em periódicos da área de enfermagem. }\end{array}$} \\
\hline \multicolumn{7}{|c|}{$\begin{array}{l}\text { Pergunta de pesquisa: "Quais as evidências científicas disponíveis na literatura sobre as características } \\
\text { necessárias para a publicação de artigos científicos em periódicos da área de enfermagem?". }\end{array}$} \\
\hline Base & Título & Autores & $\begin{array}{c}\text { Ano de } \\
\text { publicação }\end{array}$ & Objetivo & Seleção & $\begin{array}{l}\text { Motivo de } \\
\text { exclusão }\end{array}$ \\
\hline & & & & & & \\
\hline
\end{tabular}

Fonte: Autores.

Tendo em vista que a scoping review não tem objetivo de avaliar nível de evidência científica, esta análise não será realizada nesta pesquisa (Peters et al., 2015).

\section{Resultados}

A apresentação dos resultados obtidos ocorrerá primeiramente por meio de um fluxograma adaptado dos principais itens para relatar Revisões sistemáticas e Meta-análises: (PRISMA) (Page et al., 2021), o qual contemplará as fases de identificação, triagem, elegibilidade e quantidade de estudos que permanecerão em cada etapa, considerando os critérios de inclusão e exclusão.

Posteriormente, será realizada a síntese dos principais resultados de cada estudo incluído na scoping review, de forma descritiva, por meio de um quadro.

Este estudo proposto permitirá a identificação, compilação e disseminação de evidências disponíveis sobre as características necessárias para a publicação de artigos científicos em periódicos da área de enfermagem, com o desenvolvimento de uma escala que auxiliará os avaliadores de artigos científicos nesta tarefa.

Ressalta-se que este estudo será redigido seguindo os itens da lista de verificação do PRISMA Extension for Scoping Reviews (PRISMA-ScR) (Tricco et al.,2018).

\section{Considerações Finais}

A publicação dos resultados de uma pesquisa apresenta-se como recurso de universalização e internacionalização da ciência, permitindo a ampla divulgação dos achados, contribuindo para o ensino, pesquisa e prática.

As publicações precisam apresentar certas características que confiram rigor científico e qualidade ao artigo. Neste sentido, destaca-se que estudos com rigor metodológico, bem como boa redação científica, apresentam potencial superior de publicação, sobretudo em periódicos renomados e com maior fator de impacto, proporcionando maior visibilidade aos achados.

Sugere-se a elaboração e a validação de instrumentos para avaliação de artigos científicos, como forma de padronização de apreciação para avaliadores, bem como guia para redação do manuscrito para os autores.

\section{Referências}

Albuquerque, U. P. (2009). A qualidade das publicações científicas - considerações de um Editor de Área ao final do mandato. Acta Bot Bras, 23(1), 292-296. https://doi.org/10.1590/S0102-33062009000100031.

Crossetti, M. (2015). Em pauta a internacionalização e visibilidade da comunicação científica de enfermagem. Revista de Enfermagem da UFSM, 5(3). https://doi.org/10.5902/2179769219746.

Hussain, M., Rehman, R., \& Baig, M. (2020). Manuscript Writing and Publication Workshop: An Invoking Pilot Study on Enhancing Cognitive Research Capabilities in Health Sciences Institutes of Pakistan. Cureus, 12(6), e8802. https://doi.org/10.7759/cureus.8802.

Kramer, B \& Libhaber, E (2016). Writing for publication: institutional support provides an enabling environment. BMC Med Educ, $16: 115$. https://doi-org.ez78.periodicos.capes.gov.br/10.1186/s12909-016-0642-0. 
Research, Society and Development, v. 11, n. 1, e29411124688, 2022

(CC BY 4.0) | ISSN 2525-3409 | DOI: http://dx.doi.org/10.33448/rsd-v11i1.24688

Marziale, M. H. P. (2017). Editorial. Desafios da gestão editorial de periódicos científicos. Rev baiana de enfermagem, 31(3), e24028. https://doi.org/10.18471/rbe.v31i3.24028.

Pacheco, R. L, Garcia, C. M., Hosnil, N. D., Latorraca, C. de O. C., Martimbianco, A. L. C., Logullo, P., \& Riera, R. (2017). Guidelines para publicação de estudos científicos. Parte 3: Como publicar ensaios clínicos. Diagn Tratamento, 22(4), 169-75.

https://docs.bvsalud.org/biblioref/2017/11/875481/rdt_v22n4_169-175.pdf.

Page, M. J., McKenzie, J. E., Bossuyt, P. M., Boutron, I., Hoffmann, T. C., Mulrow, C. D, Shamseer, L., Tetzlaff, J. M., Akl, E. A., Brennan, S. E., Chou, R., Glanville, J., Grimshaw, J. M., Hróbjartsson, A., Lalu, M. M., Li, T., Loder, E. W., Mayo-Wilson, E., McDonald, S., McGuinness, L. A., \& Moher, D. (2021). The PRISMA 2020 statement: an updated guideline for reporting systematic reviews. BMJ, 372, n71. https://doi.org/10.1136/bmj.n71.

Pereira, M. G. \& Galvão, T. F. (2014). Etapas de busca e seleção de artigos em revisões sistemáticas da literatura. Epidemiologia e Serviços de Saúde, 23(2), 369-371.

Peters, M. D., Godfrey, C., McInerney, P., Munn, Z., Tricco, A. C., \& Khalil, H. (2020). Chapter 11: Scoping Reviews. https://doi.org/10.46658/JBIMES-2012.

Peters, M. D, Godfrey C. M., Khalil, H., McInerney, P., Parker, D., \& Soares, C. B. (2015). Guidance for conducting systematic scoping reviews. Int J Evid Based Healthc, 13(3):141-6. https://doi.org/10.1097/XEB.0000000000000050.

Ribeiro, R. P. \& Marziale, M. H. P. (2018). Editorial characteristics and quality of the articles published by Brazilian Nursing journals. Revista da Escola de Enfermagem da USP [online],52, e03367. https://doi.org/10.1590/S1980-220X2017030003367.

Tricco, A. C., Lillie, E., Zarin, W., O'Brien, K. K., Colquhoun, H., Levac, D., Moher, D., Peters, MD, Horsley, T, Weeks, L, Hempel, S., Akl, E. A., Chang, C., McGowan, J., Stewart, L., Hartling, L., Aldcroft, A., Wilson, M. G., Garritty, C., \& Straus, S. E. (2018). PRISMA extension for scoping reviews (PRISMAScR): checklist and explanation. Ann Intern Med.,169(7):467-473. https://doi.org/10.7326/M18-0850. 